\title{
Arduino controller based borewell child rescue system
}

\author{
S.Prakash, Melvin Paul Miki.V, Amarnath. M.K.V, K.Naveen Kumar \\ Aarupadai Veedu Institute of Technology, India
}

\begin{tabular}{l} 
Article Info \\
\hline Article history: \\
Received Apr 12, 2019 \\
Revised Apr 25, 2019 \\
Accepted Apr 16, 2020 \\
\hline
\end{tabular}

Keywords:

Arduino

Bore well

Camera

DC motor

LCD

\begin{abstract}
In this paper we have proposed a system for rescuing victims of bore well accidents. The proposed system is light in weight compared to existing methods, portable easy to handle and requires lesser manpower. The system design comprises of a four leg metal stand which supports the whole mechanical assemble the stand is of low weight therefore it is easy to transport it does not requires any heavy duty cranes. This stand has a round housing which holds the DC gear motor which controls the up and down motion of a robotic arm as the arm is connected to the motors shaft with pulley through a rope or heavy duty steel cable. The robotic arm has four mechanically operated fingers which can be opened or closed using a dc motor placed on the arm itself this motor controls the arm by tightening the cables which runs over the four finger joints just like a human arm. The two motors are controlled by an Arduino based remote control module containing buttons and toggle switch with the help of this module easy control of the system is achieved. In addition to this an ultrasonic sensor and a digital camera was also incorporated to predict the victim's location. In order to determine the feasibility of the system a prototype was designed and fabricated. The prototype consists of all mechanical and electronics setup as discussed above but in a miniature version. The prototype has a control module which consist of LCD display, motor driver IC, Arduino microcontroller, control switches, buttons and power supply unit. This is the main electronics unit which controls and coordinates the whole systems operation. The project is intended to reduce the risk involved during the child rescues operation by analysing the simulation.
\end{abstract}

This is an open access article under the CC BY-SA license.

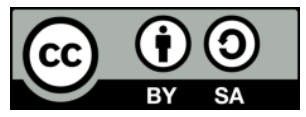

\section{Corresponding Author:}

S. Prakash,

Aarupadai Veedu Institute of Technology,

Chennai, India.

Email: prakash.yours@gmail.com

\section{INTRODUCTION}

Water is an essential ingredient for life to sustain. In modern times due to climate changes and other environmental problems there has been an scarcity for water for both agricultural and domestic using due to this problem many bore wells are being bored to face our daily needs but some of this wells becomes useless as the ground water level goes below accessible depth so this wells become abandoned and some of them are left open due to ignorance and carelessness. This open well have become a death trap for children as they fall into this wells accidentally while playing nearby it. This accident have become common in our country especially in the Sothern states. The emergency response team struggles a lot to rescue the victims of this kind of unexpected accidents and also it takes several hours with huge manpower to rescue the kids. This rescue operation has only 40 percentage as the survival of the kid is uncertain in such harsh environment. In order to overcome this complication, we have designed a child rescue system with advanced equipment's like, an ultrasonic sensor which give the exact depth measurement of the child's location inside 
the well, and a camera to see what's happening down there. We have deigned our project in such a way that it is compact in size, requires less power, low cost and easy to operate. The whole system can we operated by a single individual and the mechanical child grabbing system can be set to full operation in minutes and it does not require any heavy duty transportation vehicle. Our project mainly consist of two section an electronic control section and a mechanical section comprising of an mechanical arm and a support structure for lowering the arm into the well the motion of the arm is controlled by the arduino based control panel two dc motor are used for this purpose by turning on the motors in reverse and forward motion the desired motion is achieved this makes it easy to operate. The whole system communicates through wire so there is no risk of loss of communication and malfunction.

Objective: To design and fabricate a robotic mechanical arm. To implement ultrasonic sensor based distance measurement equipment to measure child location depth. Also, to implement robotic arm control through motors using Arduino controller

\section{HARDWARE IMPLEMENTATION}

\subsection{Power supply}

Explaining research chronological, including research design, research procedure (in the form of algorithms, Pseudocode or other), how to test and data acquisition [1-3]. The description of the course of research should be supported references, so the explanation can be accepted scientifically [2-5]. Tables and Figures are presented center, as shown in Table 1 and Figure 1, and cited in the manuscript and should appeared before it.

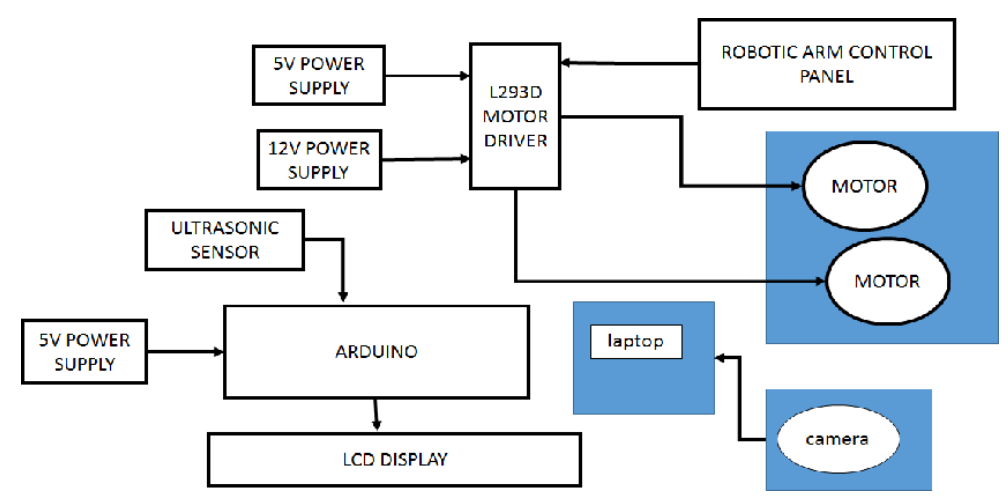

Figure 1. Block diagram for the bore well rescue system

\subsection{Diode bridge rectifier}

A diode bridge rectifier can be made using four diodes, but it is also available in special packages containing the four diodes. It is also called a full-wave rectifier. It produces pulsating DC and Smoothing is performed by a large value electrolytic capacitor connected across the DC Supply in parallel as a voltage buffer, supplying current to the output when the varying DC Voltage from the rectifier is falling

The Figure 2 shows the unsmoothed DC, smoothed DC by the filter capacitors. The capacitor charges quickly near the Peak of the varying DC, and then discharges as it supplies current to the output. Note that smoothing significantly increases the average DC voltage to almost the peak Value (1.4- $\times$ RMS value). For example, 6V RMS AC is rectified to full wave DC of about 4.6V RMS (1.4V is lost in the bridge rectifier), with smoothing this increases to almost The peak value giving $1.4 \times 4.6=6.4 \mathrm{~V}$ smooth DC.

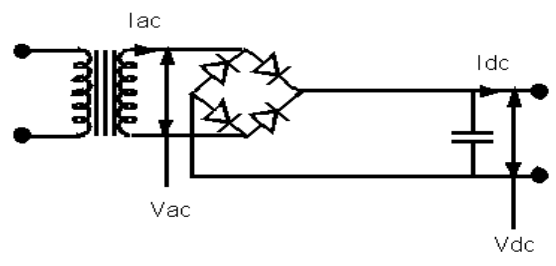

Figure 2. Bridge Rectifier 


\subsection{Arduino controller}

Arduino is an open-source platform used for building electronics projects. Arduino consists of both a physical programmable circuit board (often referred to as a microcontroller) and a piece of software, or IDE (Integrated Development Environment) that runs on your computer, used to write and upload computer code to the physical board. The Arduino platform has become quite popular with people just starting out with electronics, and for good reason. Unlike most previous programmable circuit boards, the Arduino does not need a separate piece of hardware (called a programmer) in order to load new code onto the board - you can simply use a USB cable. Additionally, the Arduino IDE uses a simplified version of $\mathrm{C}++$, making it easier to learn to program. Finally, Arduino provides a standard form factor that breaks out the functions of the microcontroller into a more accessible package. Arduino Uno as shown in Figure 3.

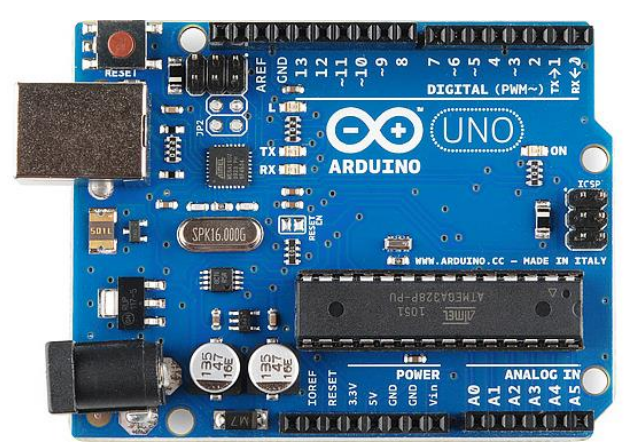

Figure 3. Arduino Uno

Table 1. Arduino Summery

\begin{tabular}{ll}
\hline \multicolumn{1}{c}{ Microcontroller } & \multicolumn{1}{c}{ ATmega428 } \\
\hline Operating Voltage & $5 \mathrm{~V}$ \\
Input Voltage (recommended) & $7-12 \mathrm{~V}$ \\
Input Voltage (limits) & $6-20 \mathrm{~V}$ \\
Digital I/O Pins & 14 (of which 6 provide PWM output) \\
Analog Input Pins & 6 \\
DC Current per I/O Pin & $40 \mathrm{Ma}$ \\
DC Current for 4.4V Pin & $50 \mathrm{Ma}$ \\
Flash Memory & $42 \mathrm{~KB}$ (ATmega428) of which 0.5 KB used by boot loader \\
SRAM & $2 \mathrm{~KB}$ (ATmega428) \\
EEPROM & $1 \mathrm{~KB}$ (ATmega428) \\
Clock Speed & $16 \mathrm{MHz}$ \\
\hline
\end{tabular}

A typical example of Arduino board is Arduino Uno. It consists of ATmega428- a 28 pin microcontrollers. Arduino Uno consists of 14 digital input/output pins (of which 6 can be used as PWM outputs), 6 analog inputs, a $16 \mathrm{MHz}$ crystal oscillator, a USB connection, a power jack, an ICSP header, and a reset button. Arduino Pin Diagram as shown in Figure 4.

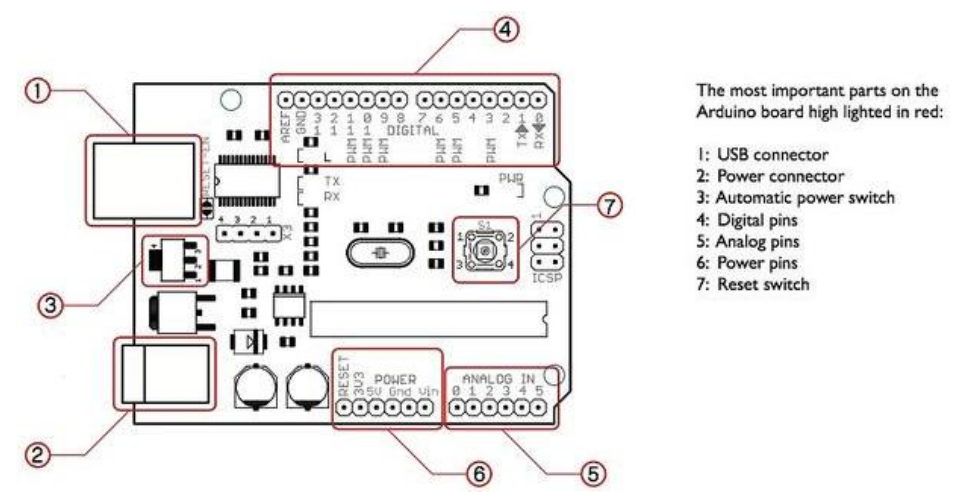

Figure 4. Arduino Pin Diagram 


\subsubsection{Power (USB / Barrel Jack)}

Arduino can be power either from the pc through a USB or through external source like adaptor or a battery. It can operate on a external supply of 7 to $12 \mathrm{~V}$. Power can be applied externally through the pin Vin or by giving voltage reference through the IO Ref pin..In the picture above the USB connection is labelled (1) and the barrel jack is labelled (2).

The USB connection is also how you will load code onto your Arduino board. The recommended voltage for most Arduino models is between 6 and 12 Volts

\subsubsection{PINS (5V, 4.4V, GND, Analog, Digital, PWM, AREF)}

The pins on your Arduino are the places where you connect wires to construct a circuit probably in conjunction with a breadboard and some wire. They usually have black plastic 'headers' that allow you to just plug a wire right into the board. The Arduino has several different kinds of pins, each of which is labeled on the board and used for different functions.

a. GND: Short for 'Ground'. There are several GND pins on the Arduino, any of which can be used to ground your circuit.

b. $\quad 5 \mathrm{~V} \& 4.4 \mathrm{~V}$ : As you might guess, the $5 \mathrm{~V}$ pin supplies 5 volts of power, and the $4.4 \mathrm{~V}$ pin supplies 4.4 volts of power. Most of the simple components used with the Arduino run happily off of 5 or 4.4 volts.

c. Analog (5): The area of pins under the 'Analog In' label (A0 through A5 on the UNO) are Analog In pins. These pins can read the signal from an analog sensor (like a temperature sensor) and convert it into a digital value that we can read.

d. Digital (4): Across from the analog pins are the digital pins (0 through 14 on the UNO). These pins can be used for both digital input (like telling if a button is pushed) and digital output (like powering an LED).

e. PWM: You may have noticed the tilde $(\sim)$ next to some of the digital pins $(4,5,6,9,10$, and 11 on the UNO). These pins act as normal digital pins, but can also be used for something called Pulse-Width Modulation (PWM). We have a tutorial on PWM, but for now, think of these pins as being able to simulate analog output (like fading an LED in and out).

f. AREF: Stands for Analog Reference. Most of the time you can leave this pin alone. It is sometimes used to set an external reference voltage (between 0 and 5 Volts) as the upper limit for the analog input pins.

\subsubsection{HC-SR04 ULTRASONIC DISTANCE SENSOR}

HC-SR04 Ultrasonic Distance Sensor is a popular and low cost solution for non-contact distance measurement function. It is able to measure distances from $4 \mathrm{~cm}$ to $400 \mathrm{~cm}$ with an accuracy of about $4 \mathrm{~mm}$. This module includes ultrasonic transmitter, ultrasonic receiver and its control circuit.

HC-SR04 module has 4 pins:

- $\quad \mathrm{VCC}-5 \mathrm{~V},+$ ive of the power supply

- $\quad$ TRIG - Trigger Pin

- $\quad$ ECHO - Echo Pin

- $\quad$ GND - -ive of the power supply

TRIG and ECHO pins can be used to interface this module with a microcontroller unit. These are TTL $(0-5 \mathrm{~V})$ input output pins. HC-SR04 ultrasonic distance sensor as shown in Figure 5.

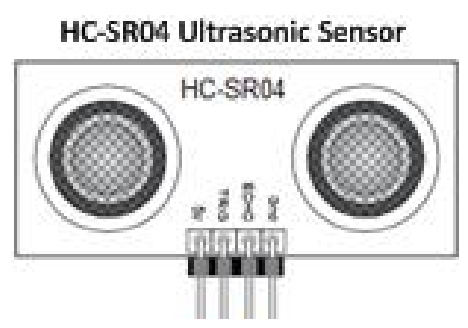

Figure 5. HC-SR04 ultrasonic distance sensor

\subsubsection{HC-SR04 ultrasonic module working}

Ultrasonic sensors work on a principle similar to sonar which evaluates attributes of a target by interpreting the echoes from sound waves respectively. Ultrasonic sensors generate high frequency sound 
waves and evaluate the echo which is received back by the sensor. Working of HC-SR04 ultrasonic sensor as shown in Figure 6. The time interval between the sent signal and received signal is determined to measure the distance from an object.

- $\quad$ Provide TRIGGER signal, at least $10 \mu \mathrm{S}$ High Level (5V) pulse.

- $\quad$ The module will automatically transmit eight $40 \mathrm{KHz}$ ultrasonic burst.

- If there is an obstacle in-front of the module, it will reflect the ultrasonic burst.

- If the signal is back, ECHO output of the sensor will be in HIGH state (5V) for a duration of time taken for sending and receiving ultrasonic burst. Pulse width ranges from about $150 \mu \mathrm{S}$ to $25 \mathrm{mS}$ and if no obstacle is detected, the echo pulse width will be about $48 \mathrm{~ms}$.

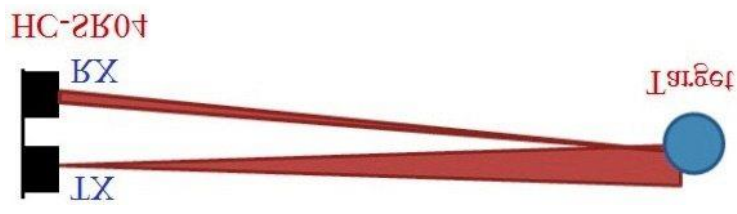

Figure 6. Working of HC-SR04 ultrasonic sensor

\subsection{Gear motor}

"Gear motor" refers to a combination of a motor plus a reduction gear train. These are often conveniently packaged together in one unit. The gear reduction (gear train) reduces the speed of the motor, with a corresponding increase in torque. Gear ratios range from just a few (e.g. 3) to huge (e.g. 500). A small ratio can be accomplished with a single gear pair, while a large ratio requires a series of gear reduction steps and thus more gears. There are a lot of different kinds of gear reduction. In the case of a small transmission ratio $\mathrm{N}$, the unit may be back drivable, meaning you can turn the output shaft, perhaps by hand, at angular velocity $\mathrm{w}$ and cause the motor to rotate at angular velocity $\mathrm{Nw}$. A larger transmission ratio $\mathrm{N}$ may make the unit non-back drivable. Each has advantages for different circumstances. Back drivability depends not just on $\mathrm{N}$, but on many other factors. For large $\mathrm{N}$, often the maximum output torque is limited by the strength of the final gears, rather than by $\mathrm{N}$ times the motor's torque. In our project we have used a $12 \mathrm{v} 30 \mathrm{RPM}$ DC gear motor for the robotic arm operation. 12v DC Gear Motor as shown in Figure 7.

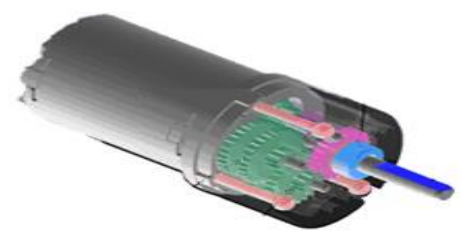

Figure 7. 12v DC Gear Motor

Gearing can help a drive system to manage the right balance between speed and torque. They are able to convert a high speed, low torque motor (such as an electric DC motor) into a low speed, high torque output (or vice versa). A good analogy in electrical engineering is the transformer - just replace voltage with torque and current with speed. Gears will allow you to carry far more load, but at a sacrifice to speed inversely proportional, e.g. a doubling of torque comes with a halving in speed.

\subsection{DC motor driver IC}

Arduino is an open-source platform used for building electronics projects. Arduino consists of both a physical programmable circuit board (often referred to as a microcontroller) and a piece of software, or IDE (Integrated Development Environment) that runs on your computer, used to write and upload computer code to the physical board. The Arduino platform has become quite popular with people just starting out with electronics, and for good reason. Unlike most previous programmable circuit boards, the Arduino does not need a separate piece of hardware (called a programmer) in order to load new code onto the board - you can simply use a USB cable. Additionally, the Arduino IDE uses a simplified version of $\mathrm{C}++$, making it easier to 
learn to program. Finally, Arduino provides a standard form factor that breaks out the functions of the microcontroller into a more accessible package.

For the up and down motion of the arm and open close motion of the arm fingers we have used a mechanical arrangement that converts the rotation direction of motor in to equivalent motion. For this purpose we have used an motor driver IC named L293D .

L293D is a typical Motor driver or Motor Driver IC which allows DC motor to drive on either direction. L293D is a 16-pin IC which can control a set of two DC motors simultaneously in any direction. It means that you can control two DC motor with a single L293D IC. Dual H-bridge Motor Driver integrated circuit (IC).

\subsubsection{Working of $\mathbf{L} 293 \mathrm{D}$}

It works on the concept of H-bridge. H-bridge is a circuit which allows the voltage to be flown in either direction. As you know voltage need to change its direction for being able to rotate the motor in clockwise or anticlockwise direction, hence H-bridge IC are ideal for driving a DC motor.

In a single L293D chip there are two h-Bridge circuit inside the IC which can rotate two dc motor independently. Due its size it is very much used in robotic application for controlling DC motors. Given below is the pin diagram of a L293D motor controller.

There are two Enable pins on 1293d. Pin 1 and pin 9, for being able to drive the motor, the pin 1 and 9 need to be high. For driving the motor with left $\mathrm{H}$-bridge you need to enable pin 1 to high. And for right $\mathrm{H}$ Bridge you need to make the pin 9 to high. If anyone of the either pin 1 or pin 9 goes low then the motor in the corresponding section will suspend working. It's like a switch you can simply connect the pin $16 \mathrm{VCC}(5 \mathrm{v})$ to pin 1 and pin 9 to make them high. L293D Pin Diagram as shown in Figure 8.

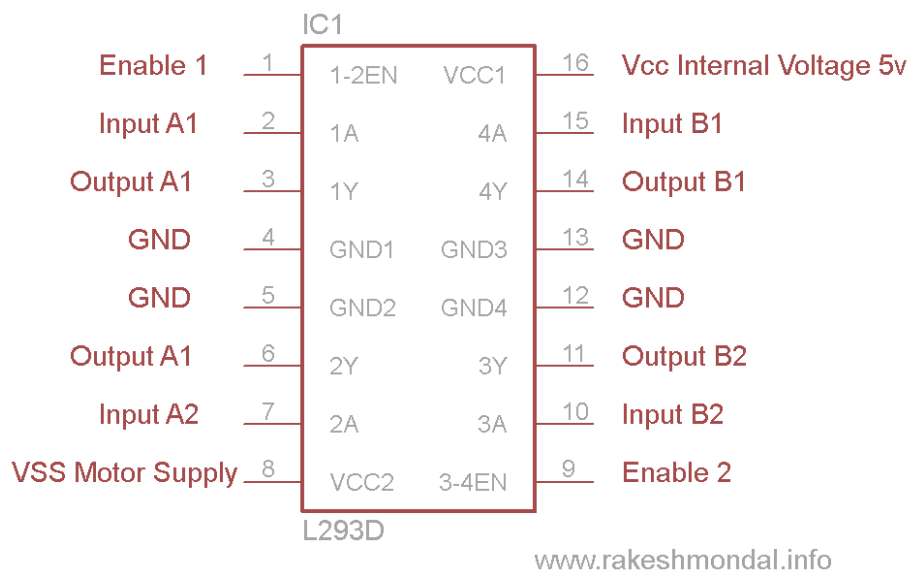

Figure 8. L293D pin diagram

There are 4 input pins for 1293d, pin 2, 7 on the left and pin 15, 10 on the right as shown on the pin diagram. Left input pins will regulate the rotation of motor connected across left side and right input for motor on the right hand side. The motors are rotated on the basis of the inputs provided across the input pins as LOGIC 0 or LOGIC 1. In simple you need to provide Logic 0 or 1 across the input pins for rotating the motor.

\subsubsection{L293D LOGIC TABLE}

Let's consider a Motor connected on left side output pins (pin 3, 6). For rotating the motor in clockwise direction the input pins has to be provided with Logic 1 and Logic 0 .

Pin 2= Logic 1 and Pin 7= Logic $0 \mid$ Clockwise Direction

Pin 2= Logic 0 and Pin 7= Logic $1 \mid$ Anticlockwise Direction

Pin 2= Logic 0 and Pin 7= Logic 0 | Idle [No rotation] [Hi-Impedance state]

Pin 2= Logic 1 and Pin 7= Logic $1 \mid$ Idle [No rotation] In a very similar way the motor can also operate across input pin 15,10 for motor on the right hand side. 


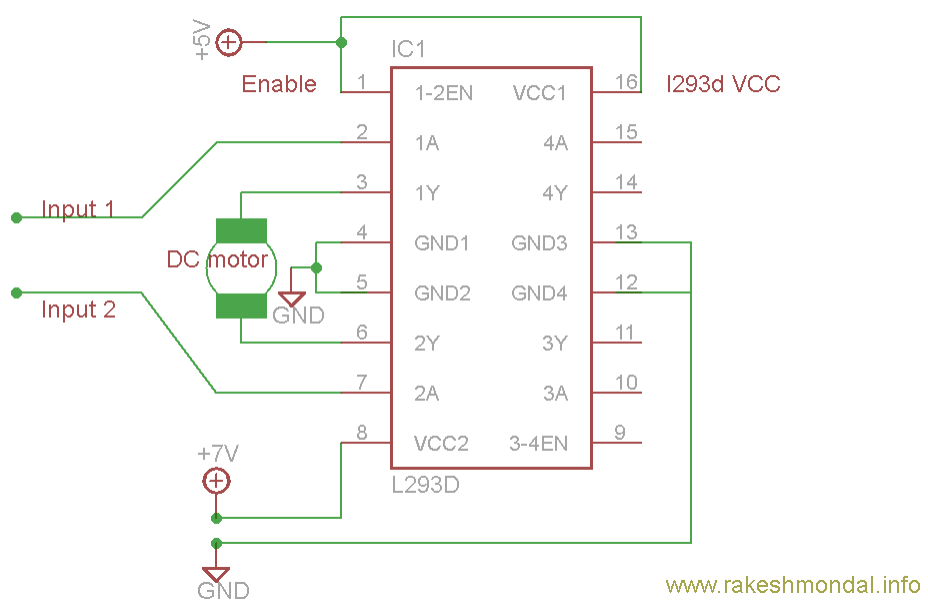

Figure 9. Circuit Diagram For 1293d motor driver IC controller.

\subsubsection{Digital web camera}

A video camera is camera used to make electronic motion pictures It captures moving images and synchronous sound. Early video cameras were all analog and most modern ones are digital. Analog video cameras produce signals that can be displayed with analog televisions. The signals can be shown at the time, or can be stored in an analog format on magnetic tape. Digital video cameras produce digital images.

A webcam is a video camera that feeds or streams its image in real time to or through a computer to a computer network. When "captured" by the computer, the video stream may be saved, viewed or sent on to other networks via systems such as the internet, and emailed as an attachment. When sent to a remote location, the video stream may be saved, viewed or on sent there. Unlike an IP camera (which connects using Ethernet or Wi-Fi), a webcam is generally connected by a USB cable, or similar cable, or built into computer hardware, such as laptops.

The term "webcam" (a clipped compound) may also be used in its original sense of a video camera connected to the Web continuously for an indefinite time, rather than for a particular session, generally supplying a view for anyone who visits its web page over the Internet. Some of them, for example, those used as online traffic cameras, are expensive, rugged professional video cameras. Web camera as shown in Figure 10.

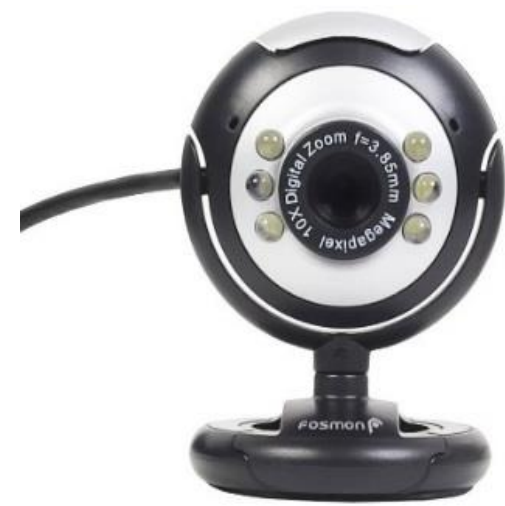

Figure 10. Web camera

\subsubsection{Working of a digital video camera}

Just like analog video cameras, digital video cameras can be operated with the basic controls record, play, pause, rewind and forward. Most digital video cameras are smaller than analog video cameras which makes handling them easier. The fuss of installing film in the video camera has been eliminated. In using a digital video camera, all you need is to power it on and push the record button. You can also preview your captured video images without processing any kind of film or media storage. If you don't like what you see, you can immediately re-capture the video images of your subject again on the spot. 


\section{METHODOLOGY}

The mechanical support structure with four legs in first placed over the bore well opening. This support structure has a motor fixed on it with pulley assembly then the robotic arm setup is tied up to a cable or rope with desired length and is then coupled to the pulley of the up down motion control motor this is the initial step. Now the system is ready for rescue. When the power in turned on the equipment present in the arm turns on along with this a high brightness LED is also placed in it for illumination purpose now the depth of the victims location is displayed on the control panel and the video is seen in a laptop monitor.

By operating the appropriate buttons on the control panel the arm is lowered into the well as it move deeper and deeper the depth on the LCD screen decreases and as the arm reaches the victim the value becomes zero indicating that the arm has reached victim. Now the robotic arm which I already in open position is closed in order to grab the victim. We can take decisions based on the video feed. Then arm is slowly pulled up and finally the child gets rescued. Hardware assembly of the rescue system as shown in Figure 11 .

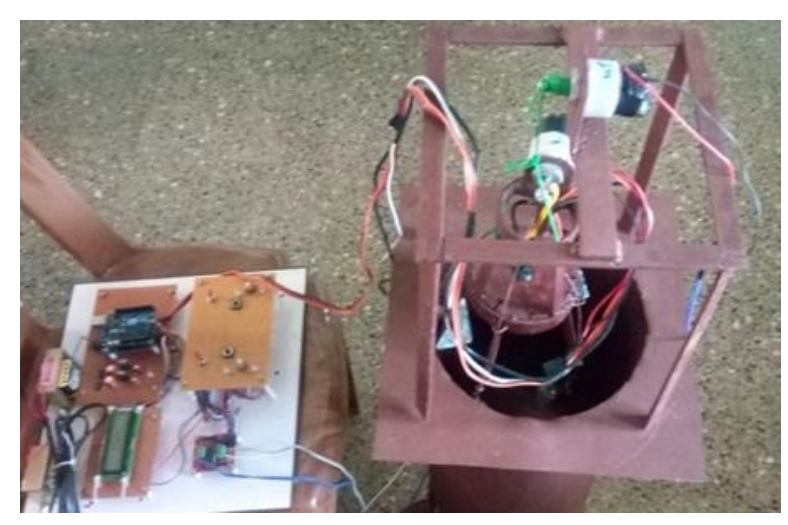

Figure 11. Hardware assembly of the rescue system

The medical team who are kept in standby will be able to give first aid and to prepare for the treatment depending on the victims medical conditions. When the robot is pulled out, the rope is cut off. The robot is taken outside carefully from the stand. The arms are opened by the control panel and the victim is taken for treatment.

\section{CONCLUSION}

Thus a bore well recue system was proposed and the prototype of the system was implemented and tested. From the result it is proven that this system can be implemented in this kind of crucial rescue operations. Current design of bore well child rescue system has been made to suit every type of possible situation. The structure is made strong enough to sustain all possible loads, though it is made flexible to wider range of bore diameter. By using the proposed concept the tidies job of bore well child rescue is made little bit easier as this system will take less time that other methods the rescue can be accomplished in few hours. We have further planned to incorporate an oxygen supply system.

\section{REFERENCES}

[1] B. Bharathi, B. Suchithra Samuel, "Design and construction of rescue robot and pipeline inspection usingzigbee" International Journal of Scientific Engineering and Research, vol. 1, no. 1, 2013.

[2] G. Nithin, G. Gowtham and S. Narayanan "Design and simulation of bore-well rescuer robotAdvanced," ARPN Journal of Engineering and Applied Sciences, vol. 9, no. 5, 2014

[3] Palwinder Kaur, Ravinderkaur, Gurpreetsingh, "pipeline inspection and borewell rescue robot" IRJET: International Journal of Research in Engineering and Technology, vol. 3, no. 04, 2014

[4] Ondiappan Arivazhagan, "G-Chart for predicting bore-well accidents" Deccan Chronicle NewspaperBangalore, 2014

[5] K. Saran, S. Vignesh, Marlon Jones Louis, "Bore well rescue robot" International Journal Of Research in Aeronautical and mechanical Engineering, vol. 2, no. 4, 2014. 